\title{
Experiencias de actividades de extensión en temas relacionados con la alimentación y su impacto en los alumnos participantes
}

Gonzalo A. Ojeda; María V. Pereyra; Adriana M. Arias Gorman; Enzo La Cava;

Melisa J. Hidalgo; Karina R. Ávalos Llano; Sonia C. Sgroppo (*)

Palabras clave: alimentos, seguridad alimentaria, nutrición, rotulado.

\section{Resumen}

La Organización Mundial de la Salud (2013) define a la salud, como el "estado de completo bienestar físico, mental y social y no la sola ausencia de enfermedad", siendo el accionar nutricional uno de los más elementales factores sanitarios, a fin de mantener buenas condiciones de salud de la comunidad. En las provincias de Corrientes y Resistencia, hay problemas serios de malnutrición en los niños y cada vez son más evidentes las enfermedades asociadas a la desnutrición y obesidad en la población. Entre los años 2006 y 2018, los docentes del Área de Bromatología del Departamento de Bioquímica de la FACENA (UNNE), desarrollaron proyectos de extensión y voluntariado, con el objetivo de aportar soluciones a esta problemática. Se realizaron actividades de capacitación nutricional y educación de la población dirigida a niños en edad escolar y adolescentes, brindando conocimientos sobre principios de nutrición, procesamiento de alimentos e higiene alimentaria y capacitando al público en general en la preparación de alimentos con importantes aportes nutricionales e interpretación del rotulado. De las actividades desarrolladas a lo largo de estos años y según las consultas realizadas, las experiencias fueron consideradas muy enriquecedoras por todos los involucrados, alumnos, docentes y representantes institucionales, permitiendo un mayor acercamiento de la universidad al medio. Se detectó una avidez de conocimientos y de interacción entre los representantes de la instituciones y la satis-

(*) Área de Bromatología. Departamento de Bioquímica. Facultad de Ciencias Exactas y Naturales y Agrimensura. Universidad Nacional del Nordeste. e-mail: sonia.sgroppo@unne.edu.ar/sonia.sgroppo@hotmail.com 
facción de los docentes de la Universidad con la tarea realizada. Para los los alumnos fue importante el aprendizaje logrado, no solo en lo académico, sino en lo social al tener la oportunidad de tomar contacto con la realidad del entorno.

\section{Introducción}

Según Taboada (2010) el rol de la extensión universitaria tiene una importante función social y es un proceso formativo integrador del vínculo universidad-sociedad. Las diversas formas de la cultura universitaria impactan fuertemente en la formación de una sociedad, contribuyendo a mejorar su calidad de vida y transformándola. La universidad debe asumir un compromiso real con la comunidad, atendiendo a las demandas, trabajando con asociaciones intermedias, sectores de la producción, instituciones públicas y privadas y la población en general. Estas actividades, deben estar basadas en la generación y transmisión del conocimiento del más alto nivel que se llevan a cabo en sus claustros.

La Organización Mundial de la Salud (2013) ha definido a la salud, como el "estado de completo bienestar físico, mental y social y no la sola ausencia de enfermedad". Es por ello, que sería imposible lograr un desarrollo sostenido de una comunidad, si se carece de la capacidad de mantener sus buenas condiciones de salud, y es aquí donde se inserta como el más elemental de los factores sanitarios, el accionar nutricional.

Las encuestas nacionales sobre el estado nutricional de la población infantil en las provincias de Corrientes y Resistencia, ubi- cadas en el NE de Argentina, han permitido vislumbrar la magnitud del problema de malnutrición de este grupo etario. Particularmente la prevalencia de niños desnutridos lleva a la necesidad urgente de formular medidas tendientes a la normalización del estado de salud de quienes, indudablemente representan el futuro de ambas provincias.

Actividades de capacitación nutricional y educación de la población dirigida a niños en edad escolar y adolescentes, a los que se les brinde conocimiento sobre principios de nutrición, procesamiento de alimentos e higiene alimentaria y capaciten al público en general en la preparación de alimentos con importantes aportes nutricionales, puede ser una herramienta válida para mejorar la calidad de vida de la población.

La alimentación de niños en edad escolar es la vía más eficiente para acercar los medios y educarlos en la sana nutrición dado que estos niños serán los adultos del día de mañana. Además, los niños tienen un efecto multiplicador de sus inquietudes en el seno de las familias y entorno social que los rodea, motivo por el cual, su instrucción es fundamental si se busca una mejora en los hábitos alimentarios.

Las enfermedades alimentarias actuales tales como la bulimia, anorexia, obesidad y desnutrición son expresiones de una misma problemática y la forma de evitar su expansión es el conocimiento. La ausencia de una adecuada nutrición hace que las desigualdades intelectuales y sociales sean cada vez más serias y marcadas, por lo tanto solo quienes sean bien alimentados podrán aspirar a un buen desarrollo personal. 
La desnutrición provoca disminuciones en las capacidades del ser humano inclusive en su voluntad, además, el riesgo de contraer enfermedades transmitidas por alimentos debe ser minimizado. Por otra parte, la obesidad debido a la ingesta de alimentos de bajo valor nutricional provoca serios inconvenientes de salud en la población.

Además del conocimiento acerca del contenido nutricional de un alimento, una herramienta de la que disponen los consumidores es su rotulado. Este es el principal instrumento con que cuenta el consumidor para informarse acerca no solo de su contenido nutricional, sino también de la presencia de sodio o sustancias capaces de dar reacciones de sensibilidad o provocar alergias (alergenos). El rótulo es el medio de comunicación más importante entre el elaborador y el cliente al respecto y la información provista en el rótulo deber ser correctamente interpretada por el lector. En numerosas ocasiones se ingieren sustancias como el sodio o sustancias alergénicas, cuyos niveles podrían exceder los límites aconsejados y provocar desórdenes en la salud del individuo.

Por otra parte, el conocimiento de las buenas prácticas de manufactura y la forma óptima de elaboración de los alimentos, es otro factor que gravita en la buena alimentación de la población. Se debe tener en cuenta que si se aplican las buenas prácticas de manufactura, se pueden evitar problemas de contaminación microbiana, reduciendo el deterioro de los alimentos y asegurar su inocuidad. Además, un correcto procesamiento de los alimentos no solo permitirá retener sus principios nutricionales, sino también prolongar su tiempo de almacenamiento.

Si bien la función de la Universidad no es resolver en forma directa los problemas sociales, sanitarios, educativos o económicos, es responsable de generar nuevas ideas que permitan hacerlo. Se debe tener presente que la tarea fundamental de los docentes es la enseñanza soportada en la investigación, además de las actividades de extensión que los involucra con la sociedad a la que pertenecen.

Por lo anteriormente expuesto, los integrantes del Área de Bromatología del Departamento de Bioquímica, buscaron acercarse a la sociedad, instruyendo a los destinatarios para que en su vida diaria puedan seleccionar los alimentos teniendo en cuenta su contenido nutricional, la presencia de sustancias riesgosas para la salud, los ingieran en condiciones óptimas, conozcan técnicas sencillas y básicas de mantenimiento, higiene y elaboración de los alimentos, disminuyendo los riesgos de adquirir alguna enfermedad transmitida por alimentos (ETA).

Asimismo se generó un espacio académico en el cual los alumnos avanzados de carreras de grado de la Facultad, Bioquímica y Licenciatura en Ciencias Químicas, puedan aplicar lo aprendido en los años de estudio previo sobre situaciones reales, guiados por el equipo docente.

Uno de los resultados esperados con estas actividades es el inicio de un cambio de hábitos alimentarios, lo cual de por si es una ardua tarea, implicando una modificación 
cultural. Si bien su efecto no será inmediato, solo a través del conocimiento se logrará un impacto, debiendo mantenerse en el tiempo para ser realmente efectivo.

\section{Desarrollo}

Desde el año 2006, los docentes a cargo del Área de Bromatología (Departamento de Bioquímica, FACENA) generaron proyectos de extensión o voluntariado universitario, a fin de aportar soluciones a uno de los problemas más serios a los que nos enfrentamos. En el período 2006-2018, se desarrollaron los proyectos que se listan en la Tabla 1.

Tabla $N^{\circ}$ 1. Proyentos realizados en el periodo 2006-2018

\begin{tabular}{|c|c|c|}
\hline Próyectó & Corvocotótila & Periodo \\
\hline Beneficios del consumo de wegetales frescos & $\begin{array}{l}\text { Uniwersidad en el } \\
\text { Medio }\end{array}$ & $\begin{array}{l}2005 \\
2007\end{array}$ \\
\hline Podemos mejorer nuestra allimentacion? & $\begin{array}{l}\text { Voluntariádo } \\
\text { Uniwersitario }\end{array}$ & 2008 \\
\hline Calidad de vida y alimentación & $\begin{array}{l}\text { Uniwersidad en el } \\
\text { Medio }\end{array}$ & 2009 \\
\hline Y si revalorizamos nuestros wegetales? & $\begin{array}{l}\text { Voluntariado } \\
\text { Uniwerştario }\end{array}$ & 2010 \\
\hline $\begin{array}{l}\text { Edućación alimghtaria y manipulación de } \\
\text { alimentos. }\end{array}$ & UNAWE Salud & $\begin{array}{l}2012 \\
2013 \\
\end{array}$ \\
\hline $\begin{array}{l}\text { Que puede hacer la Bromatologla por la } \\
\text { sociedad? }\end{array}$ & UNAE Salud & 2014 \\
\hline $\begin{array}{l}\text { Educación alimentaria y manipulación de } \\
\text { alimentos. }\end{array}$ & UNNE Salud & $\begin{array}{l}2015= \\
2017\end{array}$ \\
\hline $\begin{array}{l}\text { La alimentación, factor clave en el mejoramiento } \\
\text { de la calidad de vida de la población }\end{array}$ & $\begin{array}{l}\text { UNWE Salld + } \\
\text { Exactas } 2018\end{array}$ & 2018 \\
\hline
\end{tabular}

El objetivo general de los proyectos a lo largo de estos años fue incrementar el conocimiento de la población en temas de nutrición, elaboración, manipulación y rotulado de los alimentos, a fin de mejorar su calidad de vida a través de la educación alimentaria.

Entre los objetivos particulares se pueden mencionar: a. Capacitar a los alumnos voluntarios en temas de nutrición, elaboración, manipulación, buenas prácticas de manufactura y rotulado de los alimentos,

b. Informar, enseñar y asesorar a los destinatarios sobre la composición de los alimentos, las buenas prácticas de elaboración, higiene, correcta manipulación y 
conservación de los alimentos y su rotulado.

c. Instruir al sector de la población seleccionado acerca de la importancia de tener una dieta equilibrada y como prevenir la enfermedades transmitidas por alimentos (ETA).

d. Mejorar los niveles de ingesta de sodio y vitamina $\mathrm{C}$ de los consumidores $\mathrm{y}$ evitar el consumo de alergenos.

e. Incrementar la confiabilidad de la población en los alimentos ingeridos.

Los destinatarios de estas actividades fueron niños y adolescentes, manipuladores de alimentos de los comedores institucionales (comedores comunitarios, jardín de infantes, escuelas), padres, docentes, público en general. Las actividades se desarrollaron en escuelas de primera infancia, primarias,
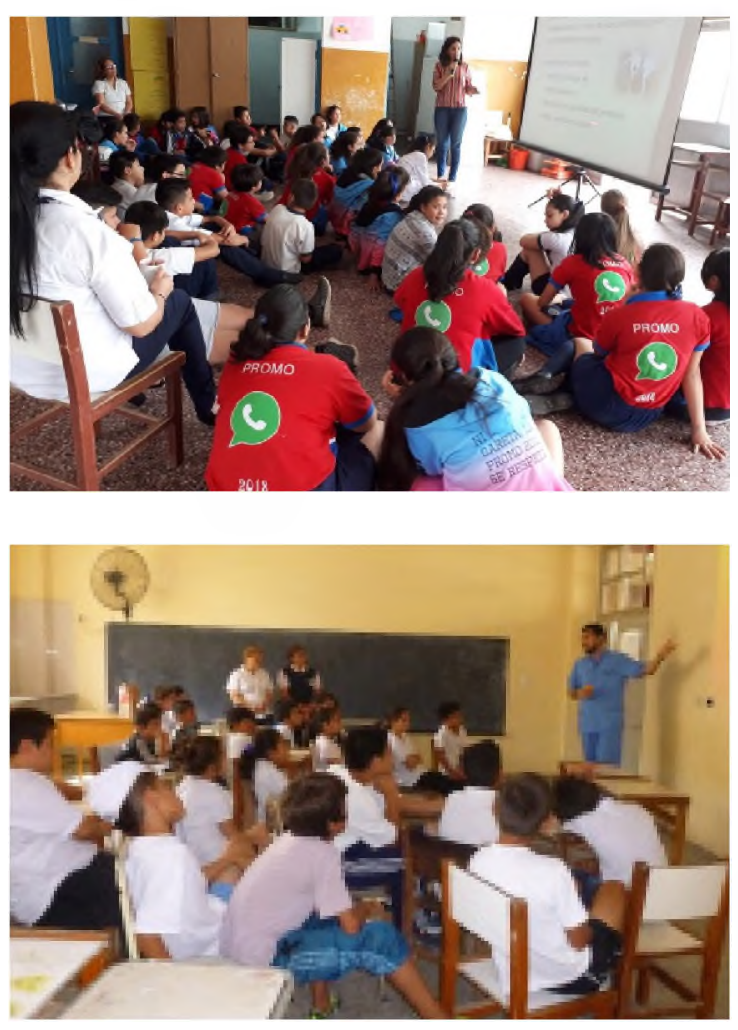

secundarias, institutos, comedores comunitarios y una unidad penal ubicadas en las ciudades de la Pcia del Chaco: Resistencia, Villa Angela, Quitilipi, La Verde y de la Pcia de Corrientes: San Luis del Palmar y ciudad de Corrientes (Figura 1).

Para llevar a cabo los talleres, se entrenó a los alumnos realizando ensayos a escala laboratorio antes de desarrollar las charlas en las que se impartían conocimientos sobre conceptos básicos de nutrición, prácticas de elaboración e higiene y seguridad alimentaria. Alumnos y docentes diseñaron y prepararon talleres de divulgación y el material de apoyo.

Los talleres desarrollados entre el 2006 al 2018 incluyeron temas acerca del beneficio del consumo de vegetales, la importancia de los compuestos bioactivos,
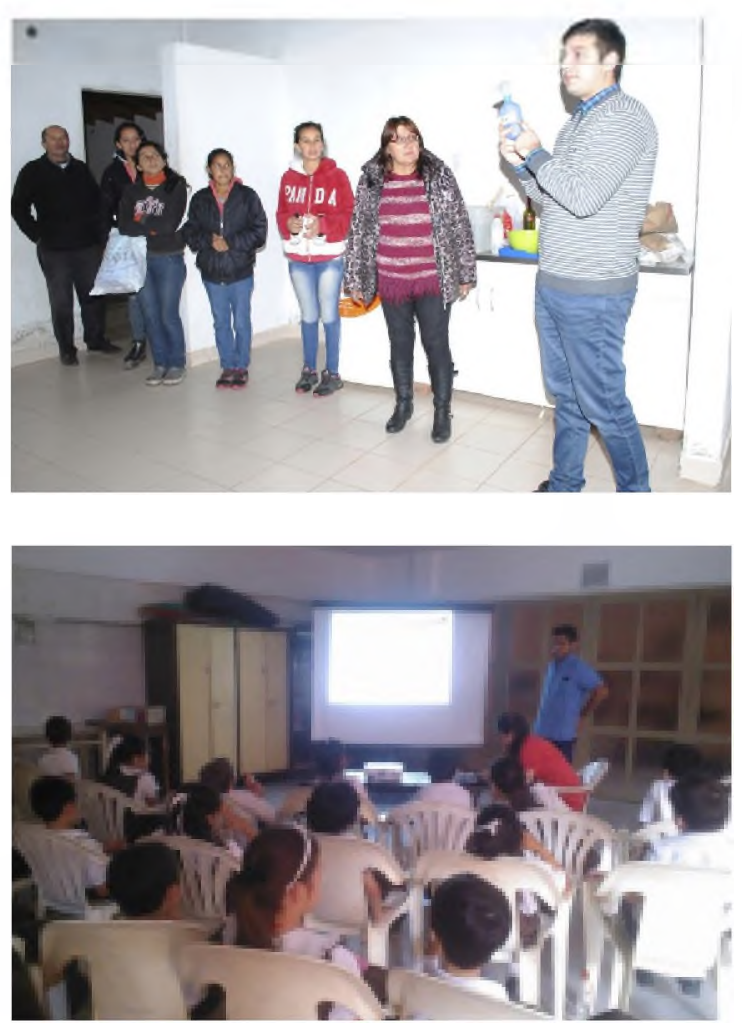

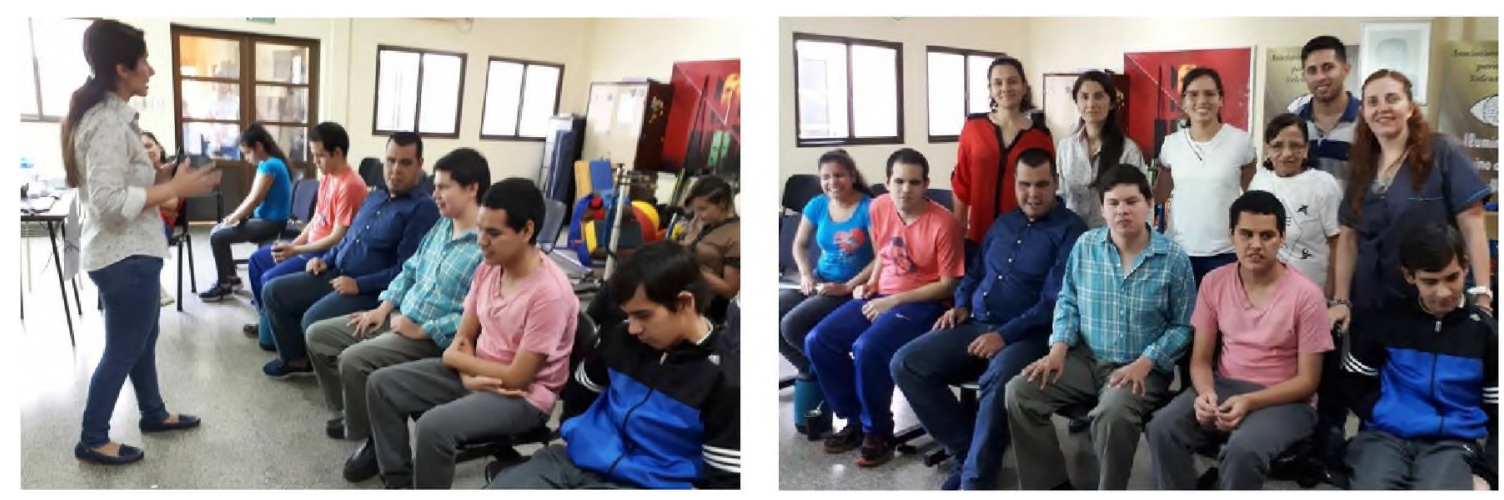

Figura 1. Registro de las actividades realizadas con diferentes destinatarios.

principios de nutrición, prácticas de elaboración de mermeladas, manipulación de alimentos, higiene y seguridad alimentaria, rotulado, los cuales fueron seleccionados por los responsables de las instituciones involucradas. posibilidad de participar fue a través de los docentes (Figura 2). Por otra parte, su principal motivación fue el interés en colaborar con la sociedad y en menor grado adquirir experiencias nuevas y certificación de las actividades realizadas (Figura 3).

\section{2-¿Cómo se enteró de estas actividades? \\ 17 respuestas}

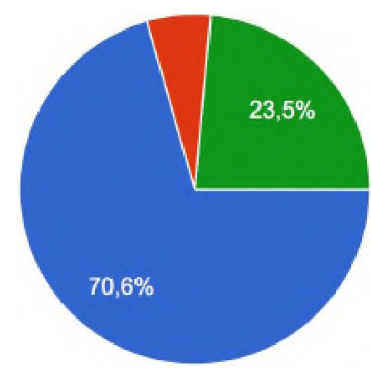

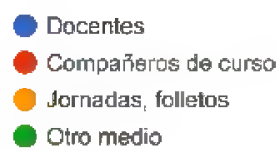

Otro medio

Figura 2. Medio de información

De acuerdo con los resultados de las encuestas realizadas a 18 alumnos de las carreras de Bioquímica y Licenciatura en Ciencias Químicas que participaron de estos proyectos, se observa que el principal medio para tomar conocimiento de la
E1 83\% (Figura 4) de los encuestados respondió que las experiencias les resultaron muy interesantes y motivadoras, y un $77 \%$ (fig. 5) opinó que eran novedosas, mientras el $22 \%$ las consideró poco novedosas. 


\section{3-¿Qué lo motivó a integrarse al proyecto?}

17 respuestas

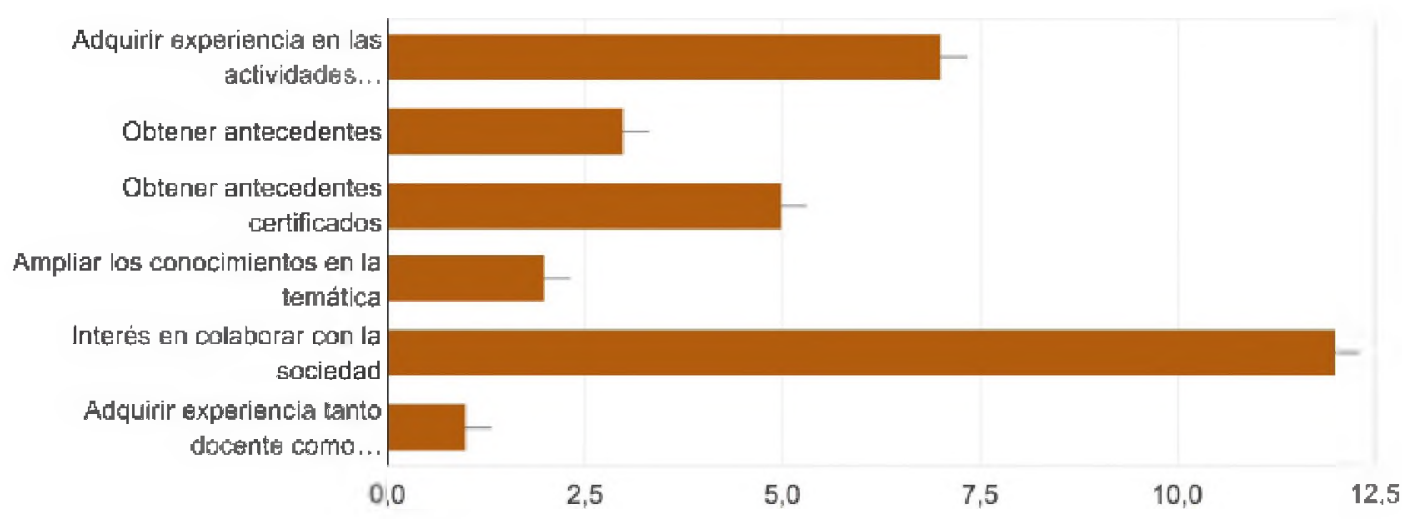

Figura 3. Motivación

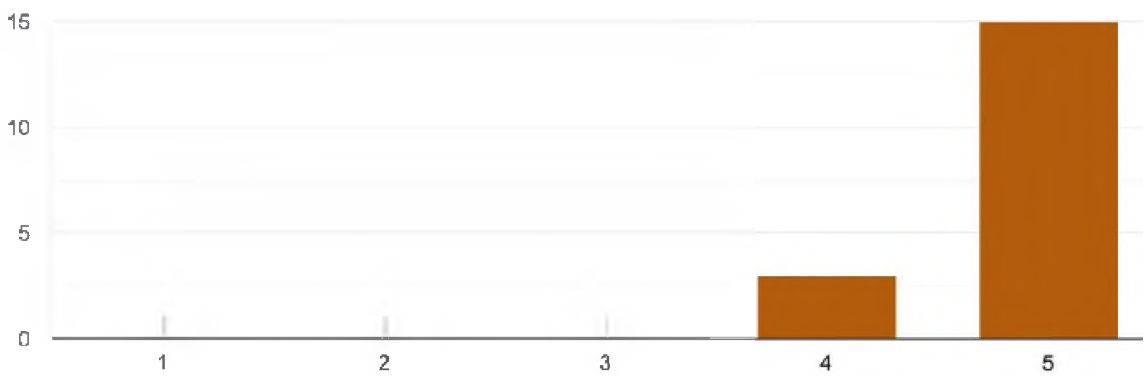

Figura 4. Motivación e interés

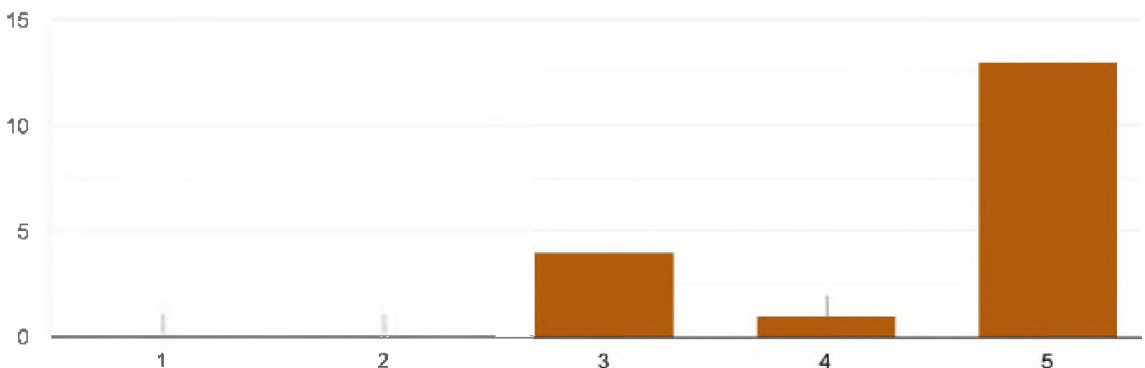

Figura 5. Novedad en las experiencias 
Asimismo, el 95\% de los alumnos piensa que las actividades tuvieron un impacto positivo mediano o muy alto en los destinatarios (fig.6) y en lo personal, el 90\% considera que fue muy beneficiado (fig. 7). Ellos consideran que obtuvieron una experiencia valiosa tanto desde el punto de vista académico, como la del desarrollo de aptitudes para integrar equipos de trabajo aprendiendo de su dinámica, como del aprendizaje de la realidad del entorno y la oportunidad de interaccionar con la comunidad. Sólo el 34\% valoró individualmente alguno de los aspectos consultados (equipo de trabajo o interacción con la comunidad) (fig. 8). Asimismo, se debe resaltar que uno de los alumnos llegó a desarrollar actividades de emprendedorismo dedicándose a la elaboración y venta de mermeladas artesanales, en base a lo aprendido durante su participación en el proyecto.

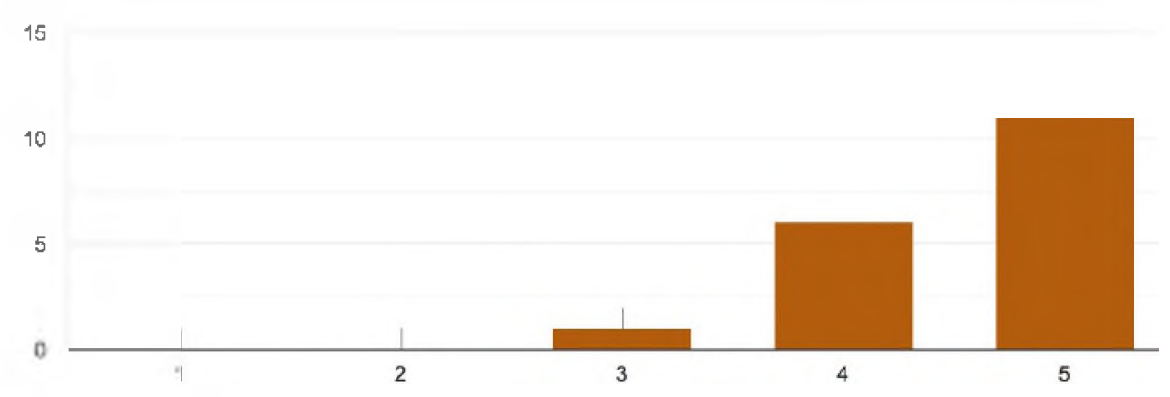

Fig. 6. Impacto en los destinatarios

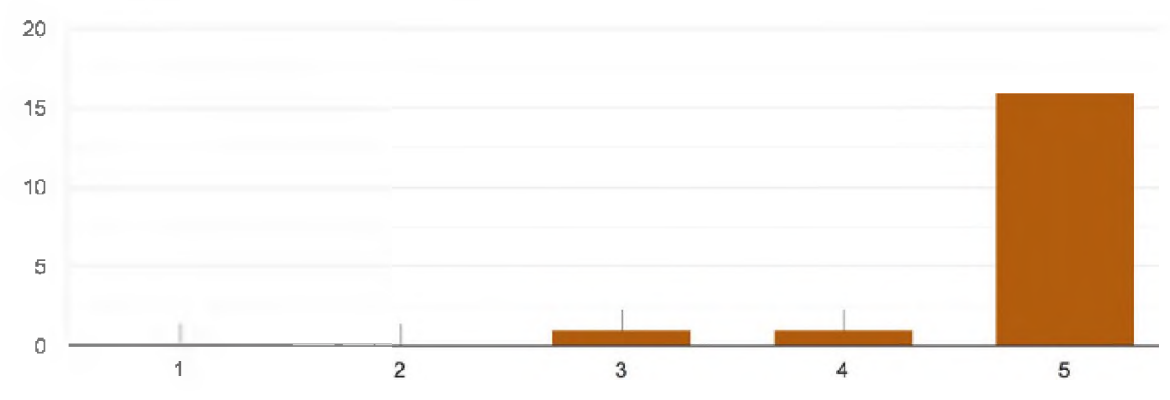

Fig. 7. Impacto en los alumnos

Se debe tener en cuenta que con estas acciones los alumnos ejecutaron acciones en beneficio de la comunidad, desarrollando una mayor sensibilidad social y ciudadana respecto de las problemáticas y necesidades de los habitantes más vulnerables en especial. Según Quezada (2015), el voluntariado universitario se transforma en un ámbito interesante para fortalecer los procesos de formación universitaria como expresión de un 
Al haber participado en el proyecto, cuáles considera son/es los/el aspecto/s más importante/s para Ud?

17 respuestas

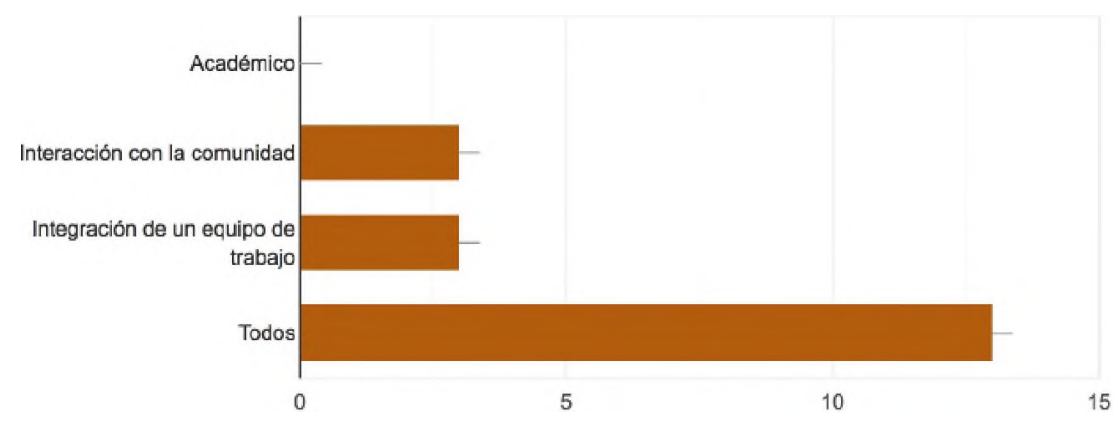

Fig. 8. Aspectos relevantes para el alumno

aprendizaje servicio. El aprendizaje servicio implica «una forma de educación experiencial en la que los estudiantes se comprometen en actividades de ayuda a la comunidad al tiempo que facilita el aprendizaje de una asignatura y el desarrollo de competencias profesionales" (Rodríguez, 2014). Con estas actividades se favorece la prosociabilidad, responsabilidad social, solidaridad, pertinencia de la actividad profesional formando en ciudadanía a los profesionales. Asimismo se favorece la vinculación con la comunidad y permite complementar la formación teórica de la universidad y detectar el potencial que tienen los alumnos como promotores de la mejora de su entorno.

Un total de 7 representantes de las instituciones participantes en los años 2017 y 2018 respondieron las encuestas, quienes en su mayoría (71\%) manifestaron que la motivación a participar de estos proyectos se debía al interés en ampliar la oferta de forma- ción, los temas los talleres y el conocimiento del grupo de trabajo, el resto tuvo interés en los temas propuestos. Asimismo, un 85\% encontró que las actividades realizadas fueron muy motivadoras, interesantes y novedosas y casi la totalidad consideró que tuvieron un impacto muy positivo en los destinatarios y en ellos mismos. A continuación se mencionan algunos de los comentarios acerca del impacto en los involucrados:

a. En los destinatarios: "mayor acercamiento a la universidad", "los alumnos aprendieron a manipular y conservar adecuadamente los alimentos", "experiencias buenas y enriquecedoras", "desarrollo de trabajos de aplicación posteriores", "lograron profundizar los temas de nutrición e higiene, aplicando a su vida cotidiana".

b. En los representantes: "la propuesta fue muy interesante dado que el tema tratado amplia los contenidos de materias teóricas y prácticas del diseño curricular", "aprender a 
transmitir conocimientos técnicos de una manera coloquial", "fue un gran experiencia de trabajo en equipo y enriquecedora a nivel social", "fue bueno, me permitió adquirir nuevos conocimientos, me sirvió para refrescar y profundizar los contenidos desarrollados en las charlas".

Con respecto a los docentes involucrados, 3 de ellos participan en estos proyectos desde el año 2006 y los 4 restantes inicialmente lo hicieron como alumnos y se incorporaron gradualmente como docentes al grupo de trabajo una vez graduados. Debe ser mencionado el hecho que todos ellos alcanzaron el grado académico máximo de doctor, en temas afines a estos proyectos. Las motivaciones para incorporarse a estos proyectos fue el interés de realizar actividades de extensionismo e interaccionar con la sociedad, además de lograr un mayor conocimiento en la temática. Las actividades les resultaron muy motivadoras e interesantes, medianamente novedosas y consideran que el impacto en los destinatarios y en lo personal fue positivo, remarcando la importancia que tuvo su participación tanto desde el punto de vista académico, como de relacionarse con la sociedad e integrar un equipo de trabajo.

\section{Conclusiones}

De las actividades desarrolladas a lo largo de estos años y según las consultas realizadas, las experiencias fueron muy enriquecedoras, permitiendo un mayor acercamiento de la universidad al medio, incrementando los vínculos entre los integrantes de la sociedad. Se detectó una avidez de conocimientos e interacción entre los representantes de la instituciones y la satisfacción de los docentes de la Universidad con la tarea realizada. Para los docentes es muy importante lograr la formación de mejores personas, profesionalmente reconocidos y compenetrados con la problemática social. De parte de los alumnos se resalta el valor que tiene el aprendizaje logrado, no solo en lo académico, sino en lo social al tener la oportunidad de tomar contacto con la realidad del entorno. De esta manera, desde la Universidad se pretende contribuir con la formación de profesionales con una mayor conciencia social, comprometidos con la solución de los problemas y transformándolos en sujetos activos de la sociedad a la que pertenecen.

\section{Bibliografía}

Taboada, P. 2010. www.perio.unlp.edu.ar/extensionenred/ accedido diciembre de 2018

Ricardo Gaete Quezada. 2015. El voluntariado Universitario como ámbito de aprendizaje servicio y emprendimiento social. Un estudio de caso. Ultima década. vol.23 no.43 . http://dx.doi.org/10.4067/S0718-
22362015000200009. Accedido diciembre 2018.

Organización Mundial de la Salud. 2013. https://www.paho.org/arg/index. php?option $=$ com_content $\& v i e w=\operatorname{article} \&$ $\mathrm{id}=28$ : preguntas - frecuentes $\&$ Itemid $=142$. Accedido en diciembre de 2018 